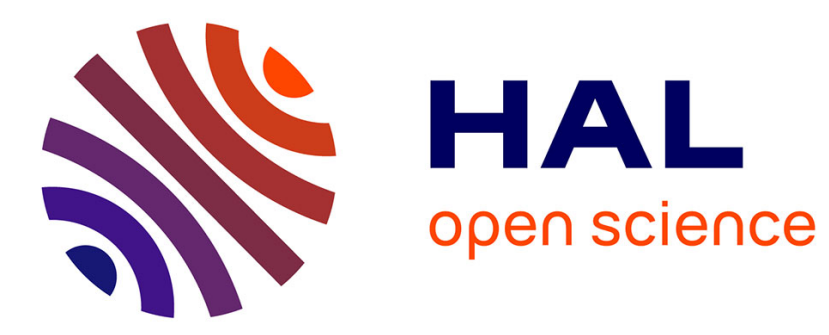

\title{
Le coût des déchets dans la dyade producteur-traiteur
} Joëlle Morana

\section{To cite this version:}

Joëlle Morana. Le coût des déchets dans la dyade producteur-traiteur. Logistique \& Management, 2005, 13 (2), pp.83-90. halshs-00259352

\section{HAL Id: halshs-00259352 \\ https://shs.hal.science/halshs-00259352}

Submitted on 27 Feb 2008

HAL is a multi-disciplinary open access archive for the deposit and dissemination of scientific research documents, whether they are published or not. The documents may come from teaching and research institutions in France or abroad, or from public or private research centers.
L'archive ouverte pluridisciplinaire HAL, est destinée au dépôt et à la diffusion de documents scientifiques de niveau recherche, publiés ou non, émanant des établissements d'enseignement et de recherche français ou étrangers, des laboratoires publics ou privés. 


\title{
Le coût des déchets dans la dyade producteur-traiteur
}

\author{
Joëlle MORANA \\ Maître de Conférences, Université d'Orléans - Laboratoire Orléanais de Gestion \\ joelle.morana@univ-orleans.fr
}

L'environnement est au cœur d'interrogations actuelles. L'axe environnemental, un des trois piliers du développement durable, impose aux entreprises une amélioration de la gestion de leurs flux, et tout particulièrement ce qui concerne les produits en retours de consommation et les déchets. Après avoir présenté les différents liens entre développement durable/axe environnement, logistique à rebours et logistique verte, l'article soumet les résultats d'une étude de terrain sur les relations entre un traiteur de déchets et les producteurs.

La loi $75-633$ du 15 juillet $1975^{1}$ définit le déchet comme «tout résidu d'un processus de production de transformation ou d'utilisation, toute substance, matériau, produit ou plus généralement tout meuble abandonné ou que son détenteur destine à l'abandon ». On dénombre à ce jour quatre catégories de déchets : les déchets ménagers et assimilés ; les déchets à risques infectieux issus, pour la majorité, des centres hospitaliers ; les déchets dangereux ${ }^{2}$ et les déchets industriels banals ${ }^{3}$. A titre d'information, le marché des déchets dangereux et banals s'élève actuellement à 6 Milliards d'Euros, à hauteur de 5,25 Milliards d'Euros pour les déchets banals et 0,75 Milliards d'Euros pour les déchets dangereux.

Cet article a pour optique d'identifier le mode de gestion des déchets d'une entreprise dont l'activité principale est la collecte et le traitement de ces derniers. En effet, "gérer un déchet » nécessite, pour chaque firme d'une chaîne d'approvisionnement, des techniques et des démarches de qualité. Néanmoins, les réglementations conséquentes en termes d'environnement n'occultent pas l'obligation pour ce type d'organisation d'être performante. Détenir le ou les indicateurs permettant de connaître la performance sociétale représente un enjeu qu'il n'est pas possible d'ignorer.
Nous présenterons tout d'abord ce qui conditionne l'importance de la collecte et du traitement des déchets dans nos sociétés actuelles. Pour cela, nous essaierons de mettre en exergue le lien entre le développement durable, la logistique à rebours ou reverse logistics, la logistique verte et la collecte et le traitement des déchets. Nous examinerons ensuite l'importance d'une «plus value informationnelle d'un indicateur » (Giard et al., 1995) dans la prise de décision des gestionnaires. Cette revue de littérature permettra d'établir une assise de réflexion dans une perspective de confrontation à une pratique de terrain. La démarche suivie juge de l'incidence d'une gestion fine pour une organisation au centre de contraintes sociétales, environnementales et économiques de plus en plus critiques.

\section{Le déchet au sein du développement durable : enjeux stratégiques d'un processus de gestion}

Bien que, comme le soulignent Krupicka et Dreveton (2005), "le développement durable ne semble pas constituer une nouvelle problématique de recherche en soi, [il] offre un terrain d'application permettant d'enrichir des problématiques de recherche anciennes (tel-
1 - Codification au titre $I V d u$ livre $V$ du code de

l'environnement.

2 - Les déchets dangereux regroupent tous les déchets issus d'une activité industrielle. Ils se présentent sous trois formes, à savoir les déchets liquides $(30 \%)$, solides $(31 \%)$ et les boues (39\%). Les principales catégories de déchets dangereux sont les déchets de cuisson, fusion, incinération pour $24,6 \%$, les déchets de traitement de dépollution et de préparation d'eau pour $23,4 \%$, les matériaux et matériels souillés $(7,6 \%)$, les déchets minéraux solides de traitements chimiques $(5,3 \%)$, les déchets de synthèse et d'autres opérations de la chimie organique $(5,2 \%)$, les déchets liquides huileux (5\%), les solvants et déchets contenant des solvants (3,5\%), les déchets minéraux solides de traitement thermiques et mécaniques $(2,5 \%)$ et les déchets de peinture, vernis, colle, mastic, encre $(1,9 \%)$.

3 - Les déchets industriels banals sont des déchets non dangereux et non inertes résultant d'une activité artisanale, commerciale, industrielle ou tertiaire. On compte parmi ces derniers le bois $(30,4 \%)$, les papiers-cartons $(29,4 \%)$, les plastiques $(6,4 \%)$, les métaux $(3,5 \%)$, le verre $(1,6 \%)$, les textiles $(0,1 \%)$ et les mélanges (28,5\%).

L'auteur remercie le lecteur anonyme pour ses remarques et suggestions sur une première version de l'article. 
les que la question de la légitimation de l'entreprise ou le changement organisationnel) tout en favorisant des réflexions tant théoriques que méthodologiques ». En général, le développement durable s'envisage au-travers de trois axes : un axe économique, un axe social et un axe environnemental. L'axe économique favorise une création de richesse pour tous par une utilisation raisonnée des ressources en milieux naturels, une évolution des relations économiques internationales, et une intégration des coûts sociaux et environnementaux dans les prix des biens et des services. L'axe social cherche à pourvoir aux besoins humains avec un objectif d'équité sociale, en vue de satisfaire les besoins essentiels des populations, lutter contre l'exclusion et la pauvreté, réduire les inégalités, et respecter les cultures. Enfin, l'axe environnemental tend à préserver, améliorer et valoriser l'environnement naturel par une gestion durable des ressources naturelles, un maintien des grands équilibres écologiques (climat, océans, etc.), et une prévention et une réduction des risques environnementaux (Depoers, 2005).

Dans ce cadre macro-économique que représente le développement durable (Krupicka et Dreveton, 2005), nous nous intéresserons plus particulièrement à l'axe environnemental. Dans cette perspective, il est possible de se reporter à Brunel (2004, p. 75) qui intègre à ce niveau " [la] biodiversité (protection et menaces, avec notamment les questions de forêts primaires, des $O G M^{4}$ ), [l']eau (gestion des bassins versants, disponibilité, qualité), [les] ressources naturelles renouvelables (faune sauvage, pêche,...), [l']épuisement des ressources fossiles, [le] problème des déchets (traitement et recyclage), [le] réchauffement climatique et effet de serre, [les] pollutions (des eaux continentales) ».

A travers ces (quelques) remarques, nous voyons dès à présent que le «problème des déchets » est un facteur essentiel dans une politique de développement durable. Or, l'enjeu du traitement et du recyclage des déchets passe, selon nous, par une réflexion en amont des stratégies des entreprises. A ce titre, il ne peut être discuté de déchets sans une connaissance des systèmes utilisés dans le cadre des logiques des chaînes logistiques. Plusieurs concepts peuvent alors être rapprochés du management environnemental : l'éco-logistique ou écologie-logistique, le soutien logistique intégré (Cassio, 1998), la logistique à rebours ou encore la logistique verte. Il serait difficile de développer ici tous ces points. Pour notre part, nous nous intéresserons à la logistique verte et à la logistique à rebours, notions qui seront alors rattachées au développement durable, puis à la gestion du déchet.

Pour Philipp (1999), la logistique verte s'apprécie à deux niveaux, à savoir la logistique au sein de la protection de l'environnement où la logistique contribue à remplir une mission prédéterminée au sein de la gestion des déchets et en représente un élément constitutif et indispensable, et la protection de l'environnement au sein de la logistique où la protection de l'environnement est perçue comme contrainte générique respectée dans des opérations concernant l'ensemble des quatre sous-systèmes logistiques (approvisionnement, production, distribution et reverse). D'une manière plus globale, Rogers et Tibben-Lembke (2001) précisent que la logistique verte consiste à faire des « efforts pour mesurer et minimiser l'impact environnemental des activités logistiques ». Quant à Murphy et Poist (2003), ces derniers avancent que la logistique verte impacte les systèmes logistiques sur treize points : le transport, l'entreposage, la manipulation des matières, l'emballage, le contrôle des inventaires, le processus de commande, l'achat, la prévision des demandes, l'implantation des bâtiments, le service client, la planification de la production, la récupération et la destruction des déchets, la manipulation des retours produits et la logistique internationale. Toutefois, la logistique verte a - selon une enquête faite par ces deux auteurs auprès de sociétés américaines et non américaines inscrites au Council of Logistics Management ${ }^{5}$ - un impact plus spécifique sur le transport, l'emballage, la récupération et la destruction des déchets.

A ce stade, il convient de souligner que certains auteurs associent la logistique verte à la logistique à rebours (Beaulieu et $a l, 1999 \mathrm{a}, \mathrm{b}$ ). Ainsi, la reverse logistics, traduite par « logistique à rebours » ou "logistique retour », s'apprécie comme "un ensemble d'activités de gestion visant la réintroduction d'actifs secondaires dans des filières à valeur ajoutée » et s'appréhende par deux types de retours. Tout d'abord, le recyclage des produits ; ensuite, les retours ou les rappels de produit vers les producteurs (exemple : produit défectueux ou infecté). Les bénéfices de la reverse logistics se présentent alors sous trois formes : des bénéfices écologiques, des bénéfices stratégiques (ex. : l'impact positif vis-à-vis de la clientèle des produits faits à base de matières recyclées) et des bénéfices 
financiers (par une gestion proactive ou une meilleure connaissance des défauts).

Pour leur part, Rogers et Tibben-Lembke (2001) distinguent la logistique verte de la logistique à rebours, bien que des interfaces communes - tout particulièrement dans la manipulation des produits (recyclage, reproduction, réutilisation des emballages) - existent (Figure 1).

En bref, la recherche d'un environnement plus sain appelle tous les acteurs de la vie quotidienne à réfléchir à leurs actes de consommation, y compris lorsque cette consommation semble être finalisée !. Depuis plusieurs décennies, le code de l'environnement est devenu très strict en termes de collecte et de traitement des déchets. Par exemple, la loi de 1975 pose les grands principes de la responsabilité du producteur de déchets dont celui du pollueur-payeur. De même, les différents amendements de cette loi soulignent que le producteur de déchets doit en assurer l'élimination et est responsable de ces derniers de la collecte à l'élimination finale. De ce fait, toute entreprise doit pouvoir justifier l'origine, la nature, les caractéristiques, les quantités, la destination et les modalités d'élimination des déchets. On peut ainsi noter parmi les amendements de la loi de 1975, et depuis le 1er juillet 2002, que seuls peuvent être stockés les déchets dits ultimes en décharge. En somme, se pose - lorsqu'on parle de déchet - toute l'importance d'une traçabilité étendue du produit, de l'extraction de la matière première à sa destruction finale.

\section{Le coût des activités de déchets}

La question de la mesure de la performance dans sa dimension environnementale prend un essor depuis ces dernières années au sein de la littérature (Arnaud, 2003 ; Christophe, 2003). Les organisations qui prétendent s'inscrire dans un développement durable « s'obligent » à une information économique, sociétale et environnementale (Depoers et al., 2003). Ainsi, la loi Nouvelles Régulations Economiques (NRE) de 2001 ainsi que ses amendements dont le décret du 20 février 2002 (Arnaud, 2003) propose d'intégrer dans les rapports économiques émis par les entreprises cotées, des données sociales et environnementales. Parmi les différents documents proposés aux entreprises pour publier un rapport environnemental, force est de remarquer la prédominance de la GRI (Global Reporting Initiative) (Capron et Quairel, 2003). Etabli vers la fin 1997, ce guide a pour mission de
Figure 1 : La relation entre développement durable et déchet.

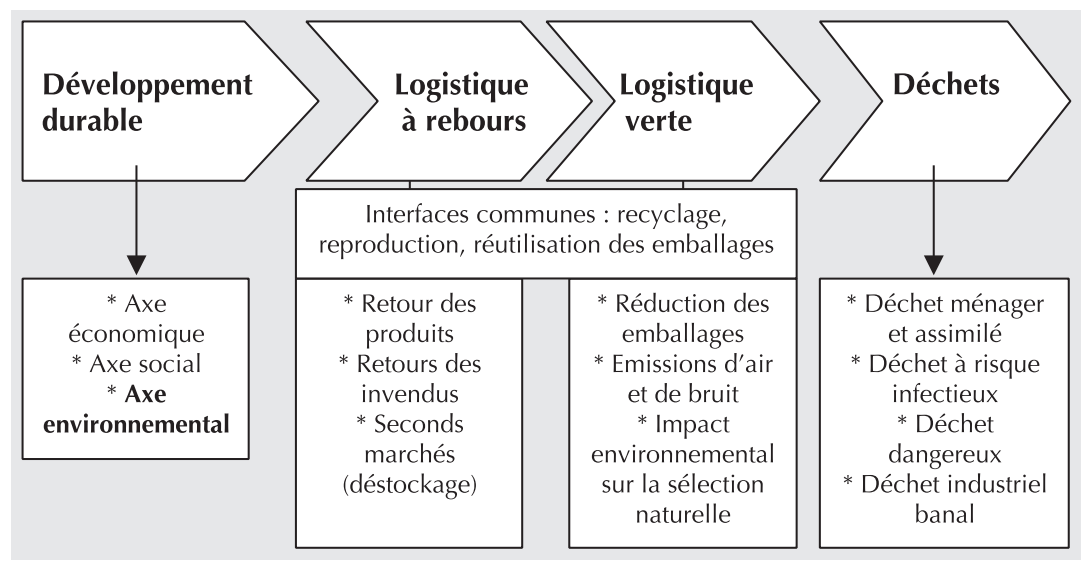

développer des directives globales en vue de rendre compte des performances économiques, environnementales et sociales. Mais, comme le remarque Quairel (2004), celui-ci nécessite des ajustements quant à une applicabilité « comptable » au sein de la firme.

De plus, différentes procédures d'audit d'environnement sont utilisées. Peuch-Lestrade (2003) en retient trois grandes catégories : l'audit de conformité réglementaire, l'audit environnement dans le cadre du commissariat aux comptes et l'audit de management de l'environnement qui se réfère plus particulièrement aux normes de types ISO 14001 ou EMAS. A ces audits s'ajoute un certain nombre d'outils et/ou approches « environnementaux » (Dreveton, 2005) :

- Le bilan environnemental, le plus ancien puisque esquissé par le Conseil National de la Comptabilité dès 1980,

- Le rapport environnement. Il présente la politique environnementale, les objectifs chiffrés (cibles), les moyens mis en œuvre, les résultats obtenus et les engagements d'amélioration en termes de politique environnementale (Antheaume, 2003),

- Le rapport développement durable. Le rapport développement durable se différencie du rapport environnement en ce sens qu'il prend appui sur un impératif de rentabilité financière dans le respect de l'environnement, des salariés et des autres parties prenantes (clients, fournisseurs, ...) (Antheaume, 2003),

- Les écobilans qui ont pour objet de comparer les résultats de l'entreprise à des normes telles que la qualité de l'air ou de l'eau,

- Les comptes verts qui affinent le plan comptable de l'entreprise et permet d'isoler les investissements liés à l'environnement. Et, en complément des comptes verts, il est possible d'établir des provisions pour ris- 
ques et charges sur des dépenses futures sur un plan environnemental (ex. : remise en état d'un immeuble dont le taux d'amiante est supérieur à la norme).

A ce titre, Christophe (2003) avait déjà présenté toute l'utilité de la mise en forme/mise en acte d'une comptabilité verte ou environnementale dont l'objet est de préciser les coûts environnementaux engendrés par l'activité de l'entreprise. Parmi ceux-ci, on peut nommer les coûts imposés (redevances ou taxes à payer), les coûts incités (investissements en environnement vs subventions étatiques) et les coûts obligatoires (remise en état d'un site après utilisation : ex. carrière). Plus intéressant ici, Christophe précise qu'une entreprise qui veut s'assurer une production en respect avec l'environnement doit effectuer des « analyses de cycle de vie » qui en amont (chez les fournisseurs), sur les sites de production et en aval démontreront que le produit "une fois utilisé, pourra être retraité sans constituer une source de pollution excessive ». Cette traçabilité du produit trouve sans contexte écho dans les démarches de logistique à rebours et de logistique verte telles que précédemment présentées.

Nonobstant, et bien que les lignes directrices de la GRI puisse apparaître comme un référentiel normatif proche des cadres prévus dans les normes ISO (Capron et Quairel, 2003 ; Quairel, 2004), Depoers (2004) note son avantage par une plus grande déclinaison d'indicateurs environnementaux. Une comparaison entre la GRI et la loi NRE met en exergue ce différentiel. Si la loi NRE propose 14 indicateurs génériques, la GRI va plus loin en énumérant pas moins de 35 indicateurs représentatifs du «volet environnemental ». Le Tableau 1 expose la partie relative aux déchets, qui nous intéresse dans cette analyse.

Tableau 1 : Les indicateurs environnementaux relatifs aux déchets (extrait : Depoers - 2004)

\begin{tabular}{|c|l|}
\hline $\begin{array}{c}\text { Loi NRE article } 2 \text { du décret } \\
\text { 2002-221 pris pour l'application } \\
\text { de l'article L225-102-1 } \\
\text { du Code du commerce }\end{array}$ & \multicolumn{1}{|c|}{$\begin{array}{c}\text { Global Reporting Initiative } \\
\text { (volet environnemental) }\end{array}$} \\
\hline Déchets & $\begin{array}{l}\text { EN11. Quantité totale de déchets par type } \\
\text { et par destination } \\
\text { EN15. Pourcentage de la masse des } \\
\text { produits vendus récupérable en fin de vie } \\
\text { utile et pourcentage réellement récupéré } \\
\text { EN31. Production, transport, importation } \\
\text { ou exportation de tous déchets jugés } \\
\text { "dangereux » au titre de la convention de } \\
\text { Bâle }\end{array}$ \\
\hline
\end{tabular}

Cette présentation faite sur la relation entre développement durable et déchet et l'évaluation de la performance de ce dernier, la suite de cet article développe le mode de gestion des déchets dans le cadre d'une entreprise dont le métier se situe sur « le cycle final du produit » à savoir le traitement et le recyclage de ceux-ci. L'intérêt est de voir les concordances (ou dissonances) entre la pratique et la théorie.

\section{La société SITA Centre Ouest, société de gestion des déchets}

La société SITA est le numéro 1 européen en matière de gestion des déchets ménagers et industriels. Nous nous intéresserons à sa filiale Centre Indre (36). Elle est spécialisée dans la collecte, le transport, le groupement, la préparation au recyclage et le traitement de tous les types de déchet valorisable ou non. Son activité comprend également l'expertise en termes de réglementations, de politiques de sécurité et de gestion de la qualité (certifications normes ISO) [Cf. Annexe 1].

Pour présenter le mode de gestion des déchets de cette entreprise, deux approches ont été prises en compte. Tout d'abord, un entretien semi-directif avec le responsable commercial de SITA Centre Indre ainsi qu'une étude de données secondaires (site Intranet, plaquettes publicitaires, présentations internes de la politique environnementale, ...) permettant de « dessiner » la cartographie de la société et des outils économiques et environnementaux (indicateurs) existants tant pour l'entreprise elle-même que pour les clients (pour rappel, nous nous situons dans une chaîne logistique à rebours, le client n'est pas le consommateur final mais le producteur). Ensuite, un deuxième entretien semi-directif et l'envoi d'un questionnaire auprès des clients utilisateurs de ces indicateurs permettent d'identifier la plus-value informationnelle de chacun de ces derniers sur le plan d'une gestion logistique et d'une démarche de développement durable.

Afin de légitimer sa présence et son action au sein de chacun de ses clients, SITA Centre Ouest propose un ensemble de mesures et de suivis qui s'inscrit dans une approche environnementale. Elle met - par Internet - à disposition de ses clients des supports d'information qui peuvent être partagés avec tous les collaborateurs du client, afin de connaître le contexte réglementaire français et, tout particulièrement, de gérer, comparer et 
optimiser les coûts et pratiques relatives à la gestion de leurs déchets. Quant à sa propre gestion, l'entreprise SITA définit sept indicateurs sur un horizon à court, moyen et long terme.

\section{Les indicateurs de performance proposés aux clients de SITA :}

Il convient de rappeler que ces indicateurs sont mis à disposition des clients de SITA via Internet. Créée depuis 2003, cette prestation payante répond, de fait, à un réel besoin de l'entreprise cliente. Ainsi, seules les firmes qui ont une activité de gestion des déchets relativement conséquente utilisent cet outil (en moyenne une benne de 5 tonnes de déchet par jour). Ce faisant, ce dernier peut toutefois être refusé par les entreprises qui détiennent un département « environnement », par exemple.

Le coût des prestations. Il s'apprécie soit sur l'ensemble des filiales du client, soit sur une filiale en particulier. Les données permettent de réaliser des études comparatives entre les différents sites afin d'identifier et d'optimiser les centres de coûts principaux. Pour chaque prestation, cet indicateur précise (par mois et an), les frais de conditionnement, de reporting, de location, de manutention, de traitement et de transport. Ce taux présente également la TGAP (Taxe Générale sur les Activités Polluantes, taxe qui est fixée par l'article 266 sexies du code des douanes et correspond à 9,15 euros la tonne et est reversée au ministère des finances).

Le taux de valorisation par type de produit et du ratio de déchet. Ces deux métriques fournissent des éléments de réflexion pour toute entreprise engagée dans une certification ISO 14000 de management environnemental. Par le biais de ces données, des axes de progrès peuvent être décidés sur deux plans : tout d'abord, accroître le pourcentage de déchet par une meilleure gestion du tri au sein de l'entreprise ; ensuite, chercher à diminuer le pourcentage déchets/matière par des pratiques de gestion améliorées (exemple : commander par fut de 200 litres au lieu de 60 litres ou bien travailler uniquement avec des fournisseurs qui récupèrent les emballages).

A eux seuls, comme nous pouvons le voir, ces indicateurs correspondent aux préconisations de la GRI quant à l'évaluation des déchets (Tableau 1). De même que le taux de valorisation par type de produit et de déchet qui s'inscrit dans l'analyse de vie du produit telle que décrite par Christophe (2003) et s'ajuste
Tableau 2 : Le tableau de bord de la société SITA - Centre Ouest

\begin{tabular}{|l|l|l|}
\hline $\begin{array}{l}\text { Les indicateurs de performance } \\
\text { proposés aux clients de SITA }\end{array}$ & \multicolumn{2}{|c|}{$\begin{array}{c}\text { Les indicateurs de performance de SITA } \\
\text { pour sa propre gestion }\end{array}$} \\
\hline $\begin{array}{l}\text { Le coût des prestations : } \\
\text { connaissance du coût par étape de } \\
\text { la gestion du déchet }\end{array}$ & $\begin{array}{l}\text { Sur le plan } \\
\text { opérationnel }\end{array}$ & $\begin{array}{l}\text { Le prix de revient Km/camion } \\
\text { Le Chiffre d'Affaires/camion }\end{array}$ \\
\hline $\begin{array}{l}\text { Le taux de valorisation par type de } \\
\text { produit et du ratio de déchet: } \\
\text { permet dans un souci } \\
\text { environnemental de recycler au } \\
\text { mieux chaque type de produit. }\end{array}$ & $\begin{array}{l}\text { Sur le plan } \\
\text { commercial }\end{array}$ & $\begin{array}{l}\text { L'évolution du chiffre d'affaires } \\
\text { L'évolution du ratio client } \\
\text { La marge brute } \\
\text { La capacité à réaliser des devis }\end{array}$ \\
\hline & $\begin{array}{l}\text { Sur le plan } \\
\text { stratégique }\end{array}$ & $\begin{array}{l}\text { La fiche de gain par client : } \\
\text { variation de la situation } \\
\text { financière n-1/n }\end{array}$ \\
\hline
\end{tabular}

autant dans une approche de logistique verte (diminution des déchets) que d'une logistique à rebours (valorisation du processus de conditionnement et de réutilisation des matières). Il convient également de souligner ici l'aptitude de l'entreprise SITA « à créer » par elle-même les indicateurs clés environnementaux puisque le second entretien mené avec le responsable commercial a souligné la méconnaissance de la GRI dans l'élaboration de leurs documents internes....

\section{Les indicateurs de performance de SITA pour sa propre gestion :}

La performance de l'entreprise s'aborde sous un triple aspect exploitation, commercial et stratégique. Sur le plan de l'exploitation, deux indicateurs sont pris en compte : le prix de revient $\mathrm{Km} /$ camion et le Chiffre d'Affaires/camion. Ces deux indicateurs sont corrélés à une logique efficiente des tournées. Ainsi, en fonction des tournées, le camion collectera un déchet au moindre kilométrage. Cette approche de rentabilité, outre la diminution des coûts, a pour intérêt de réduire la pollution de l'air et la consommation d'essence par une utilisation maximale des trajets. Sur le plan commercial, quatre indicateurs sont retenus : (a) l'évolution du chiffre d'affaires, (b) l'évolution du ratio client, (c) la marge brute et (d) la capacité à réaliser des devis. Enfin, sur un plan stratégique, des fiches de gain par client sont émises chaque année pour définir la variation de la situation financière entre l'année $\mathrm{n}-1$ et $\mathrm{n}$. Il faut noter qu'une augmentation de cette variation n'est pas nécessairement un signe «positif ». En effet, dans un souci d'innovation, SITA propose une clause de partages de gain de productivité entre SITA et son client lorsqu'il est mis en place une expertise par SITA qui permet - toutes choses égales par ailleurs - de générer des économies 
sur facture des déchets. De fait, chaque partie prenante y gagne : le client a une facture « allégée » et SITA reste rentable, voit accroît ses résultats, par son expertise.

\section{La performance apportée par .....}

... les indicateurs de performance proposés aux clients de SITA

Sur la région Centre, et avec plus de 500 clients, seules deux entreprises utilisent à ce jour ces deux indicateurs dans leur gestion. Structures relativement importantes (on compte un équipementier automobile et un établissement public à caractère industriel et commercial), elles consultent au moins une fois par mois les données proposées par SITA. Sur le questionnaire envoyé, il convient de remarquer que si les deux sociétés s'inscrivent dans une démarche de logistique environnementale, ce choix ne constitue(rait) pas un axe moteur d'un développement durable applicable à l'ensemble des acteurs de la firme (à la question : «pratiquez-vous une démarche de développement durable sur les trois axes économique/social/environnement ? »), la réponse a été négative, alors qu'elle fut positive sur la question "pratiquez-vous une démarche de logistique environnementale ?»).

De plus, les réponses montrent que, pour l'une des entreprises, la consultation de ces métriques s'effectue uniquement par le service achats et le responsable environnement, alors que la deuxième entreprise n'est intéressée que sur le plan environnemental.

Cette lecture par un ou deux services s'exprime également au travers de l'utilisation de l'expertise de la société SITA dans le cadre d'une valorisation des déchets. La « connaissance étendue » de ces indicateurs par plus d'un service du client tend à souligner une volonté quant à une diminution de la quantité de déchets produite par, par exemple, une réutilisation des emballages. Ce constat de « logistique environnementale » se perçoit aussi - pour ces deux acteurs - dans une meilleure gestion des processus logistiques ; tout en soulignant, là aussi - une recherche d'amélioration sur un plan plus étendu (production et maintenance) lorsque plusieurs services ont connaissance des résultats de ces deux indicateurs (uniquement sur la maintenance pour la seconde entreprise).

Ce faisant, ces résultats doivent être relativisés par le fait que la firme qui fait appel à la société SITA pour son expertise est « consommatrice » de toutes les formes de déchet à revaloriser (aérosol, carton, déchet industriel banal, emballage souillé, ferraille, néon, palette, peinture et pile) alors que la seconde ne traite que des déchets industriels banals, à savoir des palettes et des plastiques.

En bref, il serait possible d'affirmer que ces deux indicateurs sont d'autant plus appréciés que l'entreprise utilisatrice soit consommatrice de tous types de déchet et qu'une information multiservices est mise en œuvre.

... les indicateurs de performance pour SITA elle-même

La mise en place de ces indicateurs au sein de l'entreprise se perçoit principalement sur le plan commercial et financier. Les deux indicateurs (coût des prestations et taux de valorisation par type de produit et ratio de déchet) sont utiles sur le plan commercial. Cette prestation vient en complément du contrat initié. Néanmoins, compte-tenu de son coût, elle intéresse avant toute chose les entreprises fortes consommatrices de déchet. On pourrait donc affirmer que ces données n'ont pas de réelle influence sur une quelconque politique environnementale, voire de développement durable. Toutefois, ils peuvent constituer un élément moteur pour une amélioration des processus logistiques.

Concernant les indicateurs propres à la gestion de SITA, c'est la réduction des coûts financiers qui est privilégiée. Toutefois, en ces temps où on parle beaucoup du prix du baril de pétrole et de l'épuisement de ce dernier, les indicateurs relatifs au prix de revient $\mathrm{Km} /$ camion et au Chiffre d'Affaires/camion constituent des données non négligeables pour une gestion améliorée des transports et donc, in extenso, d'une politique environnementale.

Enfin, il est possible de dire que la fiche de gain par client qui souligne la variation de la situation financière $n-1 / n$ dans une diminution du taux de déchet a une incidence directe sur un plan environnemental et non moins négligeable sur le plan économique (réduction des charges) et social (responsabilisation des acteurs).

\section{Conclusion}

L'étude présente a eu pour objectif d'observer comment la société SITA - Centre Indre évalue son efficacité en termes de gestion des déchets, tout en la faisant correspondre à des objectifs d'une politique de développement durable. Pour cela, deux points de vue ont été 
présentés. Tout d'abord, il s'agissait de présenter les indicateurs proposés par la société SITA à ses clients. Ensuite, pour SITA elle-même, quels indicateurs étaient retenus du point de vue opérationnel et par client pour parfaire à une prise de décisions.

Dans la situation décrite, il apparaît que les prises de décision justifient la mise en place d'outils synthétiques et intégratifs tels que les tableaux de bord (Bouquin, 2001). Ainsi, un processus de coopération entre producteur et traiteur présente un intérêt indéniable. Par l'intermédiaire de quelques indicateurs clés, une « expertise horizontale progressive » voit le jour. En effet, les opérations menées sur le long terme ne peuvent fournir que des résultats satisfaisants aux yeux des différents acteurs de la chaîne émission/destruction des déchets. Mieux, il est possible d'avancer qu'une gestion (traitement et expertise) des déchets renforce une cohérence dans les politiques entreprises.

Sur les résultats du questionnaire transmis aux clients de la société SITA, outre les données déjà précédemment énoncées, un élément intéressant apparaît. En effet, une question quant à la pratique entre une logistique verte ou environnementale et une logistique à rebours était posée. Les réponses montrent qu'aucune des deux sociétés ne s'inscrit dans une logistique à rebours. Donc, faire un « amalgame » entre ces deux types de logistique comme certains auteurs le font pourrait s'avérer critique quant à une meilleure approche de décision et de gestion des flux à prendre.

En bref, lorsqu' on parle environnement dans le cadre de PMEs et d'entreprises non cotées, on se rend compte que la pratique - énonciation d'indicateurs - s'ajuste aux « normes » telles que la GRI. Autant dire que les acteurs sont à même d'apprécier des moyens utiles à gérer leur environnement dans une politique environnementale et une logistique écologique. Néanmoins, des questions restent encore en suspens. Pour quelles raisons les outils proposés aux clients par la société SITA restent-ils « cloisonnés » à quelques services du client ? De même, pour les clients non-utilisateurs de ces informations, comment gèrent-ils leur politique environnementale au titre de ceux qui détiennent un service environnement et ceux qui n'en ont pas, par exemple?

En résumé, bien que les données recueillies au-travers de cette étude de cas semblent minimes (compte-tenu du très petit échantil- lon rencontré), elles soulignent quelques points intéressants dans les pratiques environnementales actuelles, à savoir qu'au-delà d'une certaine normalisation, les acteurs ressentent par eux-mêmes la nécessité d'une responsabilité dans une qualité améliorée de la gestion de leur flux logistiques et environnementaux.

\section{Bibliographie}

Antheaume, N. (2003), Le rapport environnement/développement durable. Pourquoi publier et que publier?, Revue Française de Comptabilité, $\mathrm{n}^{\circ} 356$.

Arnaud, Ph. (2003), L'information environnementale : un enjeu à moyen terme pour toutes les entreprises, Revue Française de Comptabilité, $\mathrm{n}^{\circ} 356$.

Beaulieu, M., Martin, R. et Landry, S. (1999a), Logistique à rebours : synthèse de la littérature et typologie, Cahier de recherche $n^{\circ}$ 99-01, Ecole des Hautes Etudes Commerciales de Montréal, Québec, avril.

Beaulieu, M., Martin, R. et Landry, S. (1999b), Logistique à rebours : un portrait nord-américain, Logistique \& Management, Vol. 7, $\mathrm{n}^{\circ} 2$, pp. 5-14.

Bouquin, H. (2001), Le contrôle de gestion, Presses Universitaires de France.

Brunel, S. (2004), Le développement durable, Presses Universitaires de France.

Capron, M. et Quairel. F. (2003), Reporting sociétal : limites et enjeux de la proposition de normalisation internationale « Global Reporting Initiative », $24^{e}$ Congrès de l'AFC, Louvain.

Cassio, A.-L. (1998), La logistique : un levier du management de la protection de l'environnement, $2^{e}$ rencontres internationales de la recherche en logistique, Marseille, janvier.

Christophe, B. (2003), La comptabilité verte ou comment mieux informer pour contribuer au développement durable, Revue Française de Comptabilité, $\mathrm{n}^{\circ} 356$.

Depoers, F., Reynaud, E. et Schneider Maunoury, G. (2003), Comment mesurer la performance durable des entreprises ? Proposition d'une grille d'indicateurs, Gestion 2000, mars-avril.

Depoers, F. (2004), Reporting environnemental : une comparaison GRI/NRE, Revue Française de Comptabilité, $\mathrm{n}^{\circ} 364$. 
Depoers, F. (2005), Le développement durable dans l'entreprise, Revue Française de Comptabilité, $\mathrm{n}^{\circ} 375$.

Dreveton, B. (2005), L'outil de gestion des activités environnementales : une technique comptable ?, $2^{e}$ Journée Développement Durable, AIMS, Aix en Provence, 11 mai.

Giard, V., Boitout, V. et Bonmarchand, Ph. (1995), Apport de la simulation à la conception et l'interprétation de tableaux de bord de back office bancaire (CCP) et à la comptabilité de gestion, Papier de recherche $d u$ GREGOR, Paris I.

http://www.univ-paris1.fr/GREGOR

Krupicka, A. et Dreveton, B. (2005), Vers une tentative de compréhension du processus d'adoption des pratiques de développement durable, $2^{e}$ Journée Développement Durable, AIMS, Aix en Provence, 11 mai.

Murphy, P. et Poist R. (2003), Green perspectives and practices : a "comparative logistics" study, Supply chain management: an international journal, Vol. 8, n² 2, pp. 122-131.
Peuch-Lestrade, Ph. (2003), Audit d'environnement, Revue Française de Comptabilité, $\mathrm{n}^{\circ} 356$.

Philipp, B. (1999), Reverse logistics : les formes adéquates de coopération pour la chaîne logistique de valorisation des produits en fin de vie. Développements théoriques et approche de terrain, Logistique \& Management, Vol. 7, $\mathrm{n}^{\circ}$ 2, pp. 45-57.

Quairel, F. (2004), Responsable mais pas comptable : analyse de la normalisation des rapports environnementaux et sociaux, Comptabilité - Contrôle - Audit, Tome 10, Vol. 2, pp. 7-36.

Rogers, D. et Tibben-Lembke, R. (1999), «Reverse logistics» : stratégies et techniques, Logistique \& Management, Vol. 7, $\mathrm{n}^{\circ} 2$, pp. 15-25.

Rogers, D. et Tibben-Lembke, R. (2001), An examination of reverse logistics practices, Journal of business logistics, Vol. 22, $\mathrm{n}^{\circ} 2$, pp. 129-147.

\section{Annexe 1 : Descriptif des activités de la société SITA (source interne SITA)}

1. La collecte et le transport : En collecte sélective ou en collecte traditionnelle, en porte-à-porte ou en points d'apport volontaire

2. Le tri : Accueil des déchets recyclables issus des collectes des ménages ou des entreprises pour un tri matière par matière (plastique, verre, carton, métaux...). Concerne également le conditionnement, la préparation et le recyclage par des filières spécialisées. En aval de cette activité, SITA apporte aux industriels une prestation de négoce de leurs matières recyclables pour accéder aux meilleures conditions de marché.

3. Le compostage : Les déchets végétaux et fermentescibles sont réceptionnés sur les plates-formes de compostage où ils sont broyés, mis en fermentation puis criblée pour obtenir un compost aux normes de valorisation en agriculture et en horticulture.

4. Le stockage : Dans une logique de préservation maximale de l'environnement, I'exploitation des centres de stockage de déchets ultimes se fait en parfaite conformité avec la réglementation.

5. La gestion des déchetteries: Au sein des collectivités, les déchetteries restent un maillon essentiel du dispositif de collecte des déchets. SITA en assure I'exploitation technique ainsi que la gestion administrative.

6. L'expertise : Dans le choix de la précollecte à la commercialisation des produits valorisables, dans I'information et la mise en conformité avec les nouvelles réglementations, dans I'optimisation de la gestion des déchets et dans le démarrage de projets d'amélioration des processus. 